\title{
Inequalities on the Elements of the Inverse of a Certain Tridiagonal Matrix
}

\author{
By D. Kershaw
}

Abstract. Inequalities are obtained for the elements in the inverse of a tridiagonal matrix with positive off-diagonal elements.

During an investigation into the convergence properties of natural splines it was found useful to have bounds on the inverse of a tridiagonal matrix with positive off-diagonal elements. Matrices of this type arise in other branches of numerical analysis, in particular in the discrete analogue of certain second-order differential operators, and so it may be useful to record these results. The matrix is

$$
A=\left[\begin{array}{cccccc}
\lambda_{1} & 1-\alpha_{1} & 0 & \cdots & 0 & 0 \\
\alpha_{2} & \lambda_{2} & 1-\alpha_{2} & \cdots & 0 & 0 \\
0 & \alpha_{3} & \lambda_{3} & \cdots & 0 & 0 \\
\cdots & \cdots & \cdots & \cdots & \cdots & \cdots \\
0 & 0 & 0 & \cdots & \lambda_{n-1} & 1-\alpha_{n-1} \\
0 & 0 & 0 & \cdots & \alpha_{n} & \lambda_{n}
\end{array}\right]
$$

where $0<\alpha_{r}<1, r=1(1)_{n}$ and $\lambda_{r} \lambda_{r+1}>1, r=1(1) n-1$.

If the elements of $A^{-1}$ are denoted by

$$
a_{r s}^{-1}, \quad r, s=1(1) n
$$

then the following inequalities hold:

$$
\begin{aligned}
& 1<a_{s s}^{-1} \lambda_{s}<\mu_{s} /\left(\mu_{s}-1\right), \quad s=1(1) n \\
& 0<(-1)^{r-s} a_{r s}^{-1} \prod_{t=t_{1}}^{t_{2}} \lambda_{t}<\frac{\mu_{s}}{\mu_{s}-1}, \quad r, s=1(1) n, \quad r \neq s,
\end{aligned}
$$

where $t_{1}=\min (r, s), t_{2}=\max (r, s)$, and

$$
\mu_{s}=\min \left(\lambda_{s-1} \lambda_{s}, \lambda_{s} \lambda_{s+1}\right), \quad s=2(1) n-1,
$$

with $\mu_{1}=\lambda_{1} \lambda_{2}, \mu_{n}=\lambda_{n-1} \lambda_{n}$.

The proof is elementary and will be indicated only. The last column of $A^{-1}$ is given by the solution of the equations:

$$
\begin{aligned}
\lambda_{1} x_{1}+\left(1-\alpha_{1}\right) x_{2} & =0, \\
\alpha_{r} x_{r-1}+\lambda_{r} x_{r}+\left(1-\alpha_{r}\right) x_{r+1} & =0, \quad r=2(1) n-1, \\
\alpha_{n} x_{n-1}+\lambda_{n} x_{n} & =1,
\end{aligned}
$$

Received January 20, 1969, revised March 9, 1969.

AMS Subject Classifications. Primary 1515, 1558; Secondary 6550, 6562, 6580.

Key Words and Phrases. Tridiagonal matrices. 
(where $\left\{a_{r n}^{-1}\right\}$ has been replaced by $\left\{x_{r}\right\}$ for simplicity). Now $x_{2}$ cannot vanish, otherwise recursively from the first $n-1$ equations it would follow that

$$
x_{r}=0, \quad r=1(1) n,
$$

contradicting the final equation. Hence the first equation can be written

$$
-\lambda_{1} \frac{x_{1}}{x_{2}}=1-\alpha_{1}
$$

giving

$$
0<-\lambda_{1} \frac{x_{1}}{x_{2}}<1
$$

It will now be shown by induction that

$$
0<-\lambda_{r} \frac{x_{r}}{x_{r+1}}<1, \quad r=2(1) n-1 .
$$

Assume that these inequalities hold for $r=2(1) p-1$, so that in particular

$$
0<-\lambda_{p-1} x_{p-1} / x_{p}<1 .
$$

Now

$$
\alpha_{p} x_{p-1}+\lambda_{p} x_{p}+\left(1-\alpha_{p}\right) x_{p+1}=0
$$

and, as $x_{p} \neq 0$, this can be written, after multiplication by $\lambda_{p-1}$,

$$
-\lambda_{p-1} \lambda_{p}=\alpha_{p} \lambda_{p-1} x_{p-1} / x_{p}+\left(1-\alpha_{p}\right) \lambda_{p-1} x_{p+1} / x_{p}
$$

from which it follows that

(3) $\min \left(\lambda_{p-1} x_{p-1} / x_{p}, \lambda_{p-1} x_{p+1} / x_{p}\right)<-\lambda_{p-1} \lambda_{p}<\max \left(\lambda_{p-1} x_{p-1} / x_{p}, \lambda_{p-1} x_{p+1} / x_{p}\right)$.

Consideration of the inequalities

$$
-\lambda_{p-1} \lambda_{p}<-1, \quad \lambda_{p-1} x_{p-1} / x_{p}>-1
$$

shows that (3) can be more precisely written as

$$
\lambda_{p-1} x_{p+1} / x_{p}<-\lambda_{p-1} \lambda_{p}<\lambda_{p-1} x_{p-1} / x_{p} .
$$

The lower inequality is easily seen to be equivalent to

$$
0<-\lambda_{p} x_{p} / x_{p+1}<1,
$$

thus completing the proof of (2).

Next consider the last equation of (1) which can be written

$$
\alpha_{n} \lambda_{n-1} x_{n-1} / x_{n}=-\lambda_{n-1} \lambda_{n}+\lambda_{n-1} / x_{n},
$$

but as

$$
0<-\alpha_{n} \lambda_{n-1} x_{n-1} / x_{n}<\alpha_{n}<1
$$

it follows that

$$
0<\lambda_{n-1} \lambda_{n}-\lambda_{n-1} / x_{n}<1
$$

which can be rewritten, replacing $\lambda_{n-1} \lambda_{n}$ by $\mu_{n}$, as 


$$
1<\lambda_{n} x_{n}<\mu_{n /}\left(\mu_{n}-1\right) .
$$

It is now a simple matter to prove by induction using (2) and (4) that

$$
0<(-1)^{n-r} x_{r} \lambda_{r} \lambda_{r+1} \cdots \lambda_{n}<\mu_{n} /\left(\mu_{n}-1\right), \quad r=n-1(-1) 1 .
$$

For, if this is true when $r=p$, then

$$
0<(-1)^{n-p} x_{p} \lambda_{p} \cdots \lambda_{n}<\mu_{n} /\left(\mu_{n}-1\right),
$$

but from (2)

$$
0<(-)^{n-p-1} \frac{\lambda_{p-1} \lambda_{p} \cdots \lambda_{n} x_{p-1}}{(-1)^{n-p} \lambda_{p} \cdots \lambda_{n} x_{p}}<1
$$

and so

$$
0<(-1)^{n-p-1} \lambda_{p-1} \lambda_{p} \cdots \lambda_{n} x_{p-1}<(-1)^{n-p} \lambda_{p} \cdots \lambda_{n} x_{p}<\mu_{n} /\left(\mu_{n}-1\right),
$$

completing the induction. In an identical fashion it can be shown that the elements in the first column of $A^{-1}$ satisfy

$$
1<a_{11}^{-1} \lambda_{1}<\mu_{1} /\left(\mu_{1}-1\right), \text { where } \mu_{1}=\lambda_{1} \lambda_{2},
$$

and

$$
0<(-1)^{r-1} a_{r 1}^{-1} \lambda_{1} \lambda_{2} \cdots \lambda_{r}<\mu_{1} /\left(\mu_{1}-1\right), \quad r=2(1) n .
$$

To prove the inequalities in the general case the following equations for the elements of the sth column of $A^{-1}$ must be considered:

$$
\begin{aligned}
\lambda_{1} x_{1}+\left(1-\alpha_{1}\right) x_{2} & =0, \\
\alpha_{r} x_{r-1}+\lambda_{r} x_{r}+\left(1-\alpha_{r}\right) x_{r+1} & =\delta_{r s}, \quad r=2(1) n-1, \\
\alpha_{n} x_{n-1}+\lambda_{n} x_{n} & =0 .
\end{aligned}
$$

In order to use the previous line of argument it must be shown that neither $x_{2}$ nor $X_{n-1}$ vanish. Now if $X_{n-1}=0$, then using the last $n-s+1$ equations of (5) it would follow that

$$
x_{n}=x_{n-1}=\cdots=x_{s+1}=x_{s}=0, \quad \alpha_{s} x_{s-1}=1 .
$$

If $x_{2}=0$ the first $s+1$ equations would give the contradictory conclusion

$$
x_{1}=x_{2}=\cdots=x_{s-1}=x_{s}=0, \quad\left(1-\alpha_{s}\right) x_{s+1}=1 .
$$

Alternatively, if $x_{2} \neq 0$, then the argument used to derive (2) could be used again to prove that

$$
0<-\lambda_{r} x_{r} / x_{r+1}<1, \quad r=1(1) s-1,
$$

and the last of these inequalities contradicts (6).

Similarly, the assumption that $x_{2}=0$ will lead to contradictions, and so $x_{2} \cdot x_{n-1}$ $\neq 0$. It follows that (7) holds, and also, coming back from the $n$th equation of (5),

$$
0<-\lambda_{r+1} x_{r+1 /} x_{r}<1, \quad r=s(1) n .
$$


In particular, from (7) and (8)

$$
0<-\lambda_{s-1} x_{s-1} / x_{s}<1, \quad 0<-\lambda_{s+1} x_{s+1} / x_{s}<1,
$$

which, as $\lambda_{s-1} \lambda_{s}>1, \lambda_{s} \lambda_{s+1}>1$, are equivalent to

$$
0<-x_{s-1} / \lambda_{s} x_{s}<1 / \lambda_{s-1} \lambda_{s}, \quad 0<-x_{s+1} / \lambda_{s} x_{s}<1 / \lambda_{s} \lambda_{s+1} .
$$

Now the sth equation of (5) can be rewritten as

$$
1-\frac{1}{\lambda_{s} x_{s}}=-\alpha_{s} \frac{x_{s-1}}{\lambda_{s} x_{s}}-\left(1-\alpha_{s}\right) \frac{x_{s+1}}{\lambda_{s} x_{s}}
$$

and so

$$
\min \left(-x_{s-1} / \lambda_{s} x_{s},-x_{s+1} / \lambda_{s} x_{s}\right)<1-1 / \lambda_{s} x_{s}<\max \left(-x_{s-1} / \lambda_{s} x_{s}, x_{s+1} / \lambda_{s} x_{s}\right),
$$

and, using (9), this implies that

$$
0<1-1 / \lambda_{s} x_{s}<\max \left(1 / \lambda_{s-1} \lambda_{s}, 1 / \lambda_{s} \lambda_{s+1}\right) .
$$

If now

$$
\mu_{s}=\min \left(\lambda_{s-1} \lambda_{s}, \lambda_{s} \lambda_{s+1}\right)
$$

then (10) becomes

$$
0<1-1 / \lambda_{s} x_{s}<1 / \mu_{s},
$$

from which it follows that

$$
1<\lambda_{s} x_{s}<\mu_{s} /\left(\mu_{s}-1\right) .
$$

It remains to use (11) to translate the inequalities (7), (8) into inequalities on the elements themselves. This can be done by induction as was indicated in the case when the last column of $A^{-1}$ was considered and need not be described.

(Note Added in Proof. The conditions on $\alpha_{1}, \alpha_{n}$ can be relaxed to $0 \leqq \alpha_{1}<1$, $0<\alpha_{n} \leqq 1$, in which case $1<a_{s s}^{-1} \lambda_{s} \leq \mu_{s} /\left(\mu_{s}-1\right)$ for $s=1, n$.)

University of Edinburgh

Edinburgh

Scotland 